\title{
Clinical observation of the effect of prophylaxis on allodynia in patients with migraine
}

This article was published in the following Dove Press journal: Journal of Pain Research

\author{
$\mathrm{Na}$ Zhang' \\ Chun-Fu Chen ${ }^{2}$ \\ 'Department of Emergency Medicine, \\ Shandong Provincial Qianfoshan \\ Hospital, Shandong University, \\ Jinan 2500I4, China; ${ }^{2}$ Department \\ of Neurology, Shandong Provincial \\ Hospital, Shandong University, Jinan \\ 25002I, China
}

Objective: The clinical characteristics of migraine with and without allodynia were compared to evaluate the risk factors of cutaneous allodynia in migraine. The effects of prophylactic therapy on allodynia in patients with migraine were assessed based on the change in pain threshold after therapy.

Patients and methods: A total of 71 patients with migraine admitted to the Department of Neurology of Shandong Provincial Hospital were recruited in this study. The included patients were aged 18-70 years and did not present positive symptoms according to the nervous system examination. The variation in cutaneous allodynia was assessed for the role of classic prophylactic therapy in migraine-related allodynia, also termed as central sensitization (CS). Patients with migraine were randomized into two groups (topiramate and flunarizine groups), and the effect of drugs was evaluated by the change in cutaneous pain threshold between the two groups. Fifty-five patients were tested for pain threshold due to instrument failure. Pressure allodynia was measured with a force gage, and pricking was measured with Electronic von Frey Anesthesiometer. The pain threshold was measured every 3 months for 6 months. The variations in pain threshold after treatment were compared using $t$-test and $\chi^{2}$ test.

Results: Allodynia was seen in $70.4 \%$ of the patients; of these, $76.3 \%$ were females. Female gender, duration of illness, and frequency of migraine attacks per month were significantly associated with allodynia. The rate of allodynia and frequency of headache declined significantly, the number of patients with allodynia was reduced, and the pain threshold improved dramatically after treatment. The composite adverse events were low after treatment with topiramate and flunarizine. Conclusion: Allodynia was observed, especially in females who experienced frequent migraine attacks for a prolonged period. Gender, duration of illness, and number of migraine attacks per month were the major predictors of allodynia. Topiramate and flunarizine administered orally for a short duration can effectively improve the pain threshold and CS of patients with migraine, and efficiently relieve allodynia.

Keywords: migraine, prophylaxis, topiramate, flunarizine, allodynia, central sensitization

\section{Introduction}

Migraine is a complex neurovascular disorder that affects $12 \%$ of the general population $^{1-3}$ and is characterized by intense headache that might be accompanied by nausea and sensitivity to light (photophobia) and sound (phonophobia). It causes severe disability and work $\operatorname{loss}^{4}$ in a significant proportion of population during the maximum productive time of life.

Migraine patients experience an increased sensitivity of the skin with respect to common daily activities, such as combing of hair, taking a shower, shaving, or wearing

$\mathrm{Na}$ Zhang

Department of Emergency

Medicine,Shandong Provincial Qianfoshan

Hospital, Shandong University, No.

16766, Jingshi Road, Jinan 250014, China

Tel +86 I58 6527 77II

Email zn804@I63.com 
earrings, during attacks. ${ }^{5,6}$ The sensation of pain in response to stimuli that are not normally painful is known as allodynia. The prevalence estimate of allodynia in migraine patients ranges from $50 \%$ to $80 \% .^{7}$ Allodynia in migraine patients may represent clinical correlation with central sensitization (CS) ${ }^{8,9} \mathrm{CS}$ is speculated as a physiological response underlying allodynia in migraine patients. ${ }^{10}$ Previous studies have also demonstrated that CS plays a key role during migraine. ${ }^{8,9}$

Burstein et al demonstrated that second-order neurons in the caudal trigeminal nucleus or third-order thalamic neurons were involved in the genesis of CS and allodynia that determines the final clinical symptoms. ${ }^{8}$ The development of allodynia is associated with the development of resistance to treatment, especially triptans. ${ }^{6}$ Louter et al ${ }^{11}$ suggested that cutaneous allodynia was a predictor of migraine chronicity. Early treatment of migraine must be considered as the best strategy for preventing the appearance of allodynia or reducing the risk of migraine chronicity. ${ }^{7}$ Herein, we selected two kinds of prophylactic drugs: flunarizine and topiramate.

Early prophylactic therapy is essential in patients with frequent migraine attacks. Among the chronic disorders with episodic attacks, migraine and epilepsy share several features. ${ }^{12}$ These highly comorbid disorders have common genetic underpinnings. ${ }^{13,14}$ Both are considered as disorders of neuronal hyperexcitability that result in episodic attacks of neurological dysfunction, which can be partially prevented by antiepileptic drugs. ${ }^{12}$ Topiramate blocks both calcium and sodium channels, inhibits the excitatory glutamatergic receptors, and enhances the inhibitory activity of gamma amino butyric acid. ${ }^{15}$ Additionally, topiramate inhibits the central activation of the trigeminal nucleus caudalis and upper spinal cord, ${ }^{16}$ followed by the suppression and blockage of pathophysiological allodynia, thereby decreasing the CS and relieving allodynia.

Calcium channel blockers suppress the cortical spreading depression by inhibiting the calcium influx, reducing the glutamate release, and inhibiting the 5HT release. ${ }^{17}$ Subsequently, allodynia is relieved and CS reduced. Several controlled clinical studies have shown that flunarizine is effective in migraine prophylaxis. ${ }^{18}$

A majority of the prophylactic anti-migraine drugs have been evaluated for their effect on the reduction of frequency and severity of headache. However, only a few reports have addressed the effects of prophylactic therapy on allodynia in patients with migraine. Thus, the present study aimed to evaluate the therapeutic effects via altered pain threshold before and after therapy and establish the correlation between allodynia and clinical characteristics of patients to prevent cutaneous allodynia, chronic headaches, and reduce disability and complications.

\section{Patients and methods \\ Patients}

\section{Inclusion criteria}

A total of 71 patients with migraine admitted to the Department of Neurology of Shandong Provincial Hospital from June 2015 to December 2016 were recruited in this study. Migraine was diagnosed according to The International Classification of Headache Disorders II criteria. ${ }^{19}$ The included patients were aged 18-70 years and did not present positive symptoms according to the nervous system examination. All patients signed the informed consent. This research was approved by the Ethical Review of Medical Research on Human Being of Shandong Provincial Hospital affiliated to Shandong University.

\section{Exclusion criteria}

Patients with other types of primary or secondary headaches were excluded. In addition, those with severe cardiac, renal, or liver diseases, blood circulation or connective tissue diseases, or cancer were excluded. Women on oral contraception, pregnant women, and lactating women were also excluded. Patients with a somatization disorder, mental disease, chronic disease, or a history of chronic diseases were excluded. Also, patients who refused to receive treatment were not included.

\section{Measurements}

The patients' gender and age, duration of migraine, the age of onset of migraine, and frequency and severity of headache were recorded. The severity of headache was measured using the visual analog scale: mild (0-3), moderate (4-6), or severe (7-10). The duration of headache during the attack was scored as follows: $>2$ days, scored as $6 ; 12$ hours to 2 days, scored as 4; and $<12$ hours, scored as 2 . During the headache attacks, cutaneous allodynia was determined with Allodynia Symptom Checklist (ASC) questionnaire and the severity was scored as none (0-2), mild (3-5), moderate (6-8), and severe (>9). ${ }^{6}$ The patients were divided into cutaneous allodynia (CA) and noncutaneous allodynia (non-CA) groups according to the scores.

\section{Pain threshold}

The pressure pain was tested using Force One FDIX Digital Force Gage (Wagner Instruments, Greenwich, CT, USA), whereas the prick pain was tested using Electronic von Frey Anesthesiometer (IITC Life Science Co., Woodland 
Hills, CA, USA). Both pains were tested in four bilateral skin areas on the head and neck (forehead, temple, parietal area, and posterior neck) three times with 10-second intervals (Figure 1). The pressure pain was defined as the amount of pressure required to change the sensation of pressure to that of pressure and pain. We compared the change in pain thresholds every 3 months after treatment. The patients were randomly divided into two groups using random sequence table: one group with 35 patients were administered topiramate (25 mg; Xi'an-Janssen, Beijing, China), whereas the other with 36 patients were administered flunarizine ( $5 \mathrm{mg}$; Xi'an-Janssen). The dose of drugs was increased gradually from the lowest dose. The patients were given ibuprofen $200 \mathrm{mg}$ but not exceeding $400 \mathrm{mg}$. Also, they were not allowed to administer any other medication for migraine.

The duration of the treatment was 6 months with a followup visit every 3 months. The participants were requested to visit the Department of Neurology at least every 3 months to record the frequency and severity of headache, duration of migraine, pain threshold (pressure pain and prick pain), and side effects of the drugs.

\section{Statistical analysis}

Statistical analysis was performed using SPSS17.0 software (IBM, Armonk, NY, USA). Data were expressed as mean \pm SD. The normal distribution of quantitative measures was checked using the SPSS17.0 software. Statistical significance of the differences between the groups for enumerated or measurement data was analyzed using the $\chi^{2}$ test or the independentsample $t$-test. A comparison between the variables before and after drug administration was made using the paired-sample

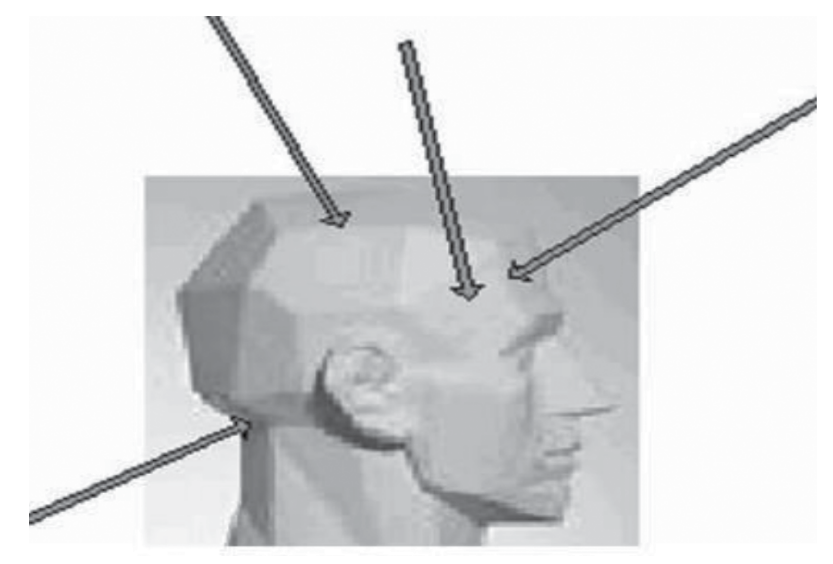

Figure I Tested areas of pain threshold.

Note: Copyright (2018 Springer Nature. Reproduced from: Filatova E, Latysheva $\mathrm{N}$, Kurenkov A. Evidence of persistent central sensitization in chronic headaches: a multi-method study. J Headache Pain. 2008;9(5):295-300. ${ }^{25}$ $t$-test. The results were considered statistically significant if $P<0.05$. The data were illustrated using GraphPad Prism Software (GraphPad Software, La Jolla, CA, USA).

\section{Results \\ Clinical characteristics}

The average age of the 71 patients with migraine was $37 \pm 13.0$ years, and 54 (76.1\%) were females. Forty-four cases $(61.9 \%)$ presented with moderate headache, 23 (32.4\%) had severe headache, $50(70.4 \%)$ had a high frequency of disease ( $>5$ headache attacks per month), and $30(42.2 \%)$ had headache for a duration of $>48$ hours.

\section{Conditions of allodynia}

According to the ASC scale scores, 50 patients $(70.4 \%)$ with migraine also presented with allodynia. The mean duration of headache was $80.8 \pm 97.2$ (range, 6-240) months. Statistical significance was not observed with respect to age (36.59 \pm 12.78 vs $40.33 \pm 14.15$ years; $t=1.124 ; P=0.265)$ and family history ( 7 vs 11 cases; $\chi^{2}=1.185 ; P=0.064$ ) between the CA and non-CA groups.

There were significantly more female patients in the CA group than in the non-CA group ( 27 vs $11 ; \chi^{2}=8.018$; $P=0.005)$. The disease duration was obviously prolonged (80.87 \pm 97.27 vs $39.95 \pm 57.06$ months; $t=2.229 ; P=0.025)$ and the frequency of headache was greater in the CA patients as compared to the non-CA patients (scores: $5.28 \pm 1.29$ vs $3.75 \pm 1.75 ; t=1.306 ; P=0.006)$. The duration of headache was prolonged in the $\mathrm{CA}$ group compared with the non-CA group (scores: $4.46 \pm 1.80$ vs $2.07 \pm 1.32 ; t=4.413 ; P=0.0001$ ). However, no significant difference was found in the severity of migraine between the two groups (scores: 6.78 $\pm 2.04 \mathrm{vs}$ 5.60 $\pm 2.29 ; t=1.076 ; P=0.273)$.

\section{Change in the numbers of patients}

The number of patients with thermal allodynia, static mechanical allodynia, or dynamic mechanical allodynia was greatly reduced after drug administration (Figure 2).

\section{Change in the pain threshold}

A total of 55 patients were tested for pain threshold due to instrument failure. Subsequently, they were randomized into two groups: 27 were administered topiramate and 28 received flunarizine.

No statistically significance difference was observed between the two groups with respect to gender (females: 18 vs 17 ; males: 9 vs $\left.11 ; \chi^{2}=0.016 ; P=0.35\right)$ and age $(35.7 \pm 12.3$ vs $38.8 \pm 13.4$ years; $t=0.794 ; P=0.265$ ). 


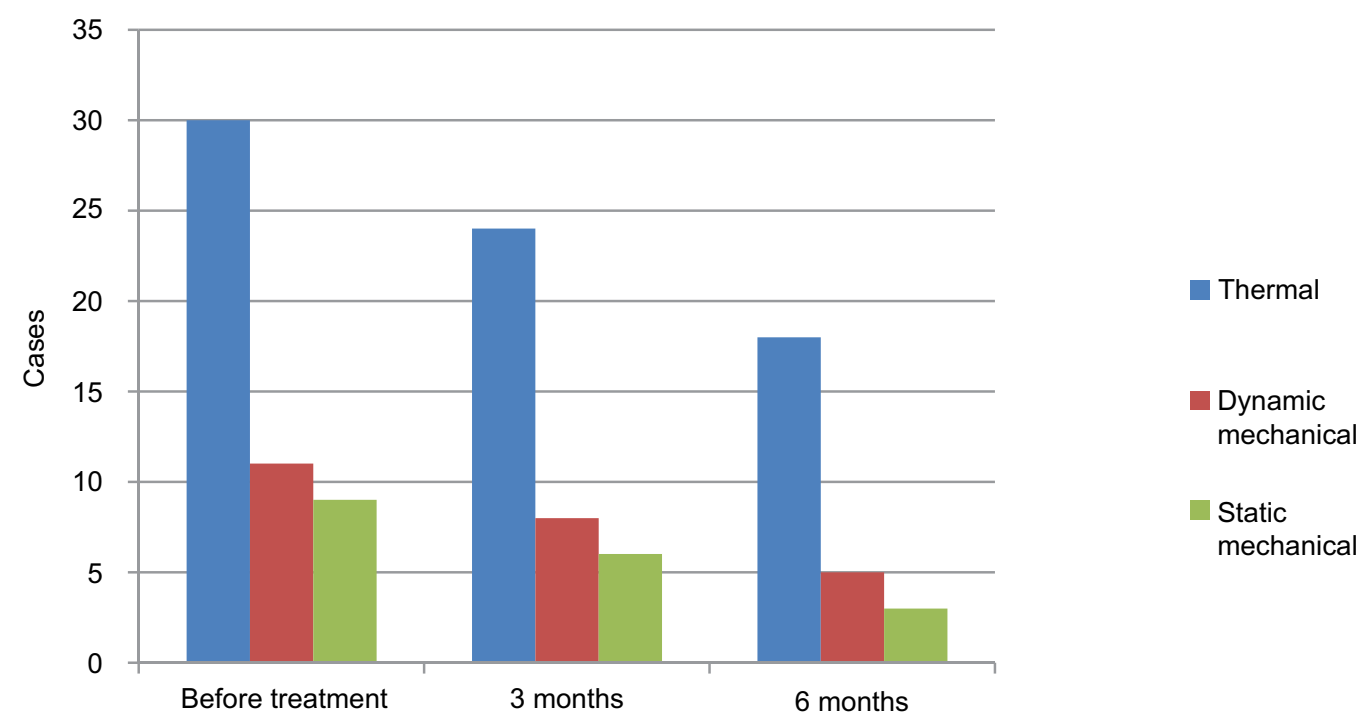

Figure 2 Changes in different types of allodynia after treatment.

The differences in the pressure pain and prick pain thresholds before and after 3 and 6 months of treatment and differences in the scores after 3 and 6 months of treatment were compared (Figures 3-6).

Furthermore, no significant difference was observed between the two groups of migraine patients with respect to pain threshold before therapy and after 3 and 6 months of therapy (Table 1).

\section{Adverse events}

In the topiramate group, three patients showed adverse events of dizziness and nausea, one patient showed loss of body weight, and three patients had slight paresthesia; the rate of incidence of side effects was $22.2 \%$. In the flunarizine group, five patients showed an increase in body weight and one had depression; the rate of incidence of side effects was $21.4 \%$.

\section{Discussion}

The present study demonstrated that $\sim 70.4 \%$ of the recruited patients developed cutaneous allodynia. The quantitative sensory testing used by Burstein et $\mathrm{al}^{8}$ revealed that $79 \%$ of the migraineurs exhibited cutaneous allodynia during headache attack. This demonstrated a high prevalence of cutaneous allodynia among migraine patients, thereby emphasizing the importance of clinical diagnosis and treatment of allodynia.

We found that cutaneous allodynia was more frequent in female patients, which has also been reported previously. ${ }^{8,20}$ This observation can be attributed to the effects of gonadal hormones on central pain modulation that can alter the pain thresholds of the trigeminal neurons. This phenomenon suggested that estrogen withdrawal in the menstrual phase can increase the activity of excitatory amino acid receptors or decrease the cutaneous stimulus threshold, leading to a wider area of allodynia. Moreover, the pain thresholds of females were speculated to be lower to noxious stimuli.. ${ }^{21,22}$

In the current study, we compared the $\mathrm{CA}$ and non-CA groups and found that with prolonged disease duration, the migraine attacks were frequent, and the duration of each migraine attack was longer with frequent prevalence of allodynia. Among migraine sufferers, allodynia is commonly reported in individuals with frequent headaches ${ }^{6}$ and extended duration of the disorder, ${ }^{23}$ suggesting that it is due to repeated sensitization of the central pain pathways over a period. As the disease duration is prolonged, the threshold of neuronal activation may change with time and repetitive sensitization of the central pathways may cause allodynia.

Frequent migraine in patients is usually evolved from episodic attacks. The potential pathogenic mechanism underlying this phenomenon is that pain pathways are chronically sensitized from repeated episodes of headache attacks. ${ }^{24,25}$

The phenomenon of sensitization results in a corresponding reduction of the activation threshold, an increased neuronal reactivity, and an expansion of the receptive fields related to second-order neurons. The presence of allodynia is speculated to be a predictor of chronic migraine. ${ }^{6}$ 


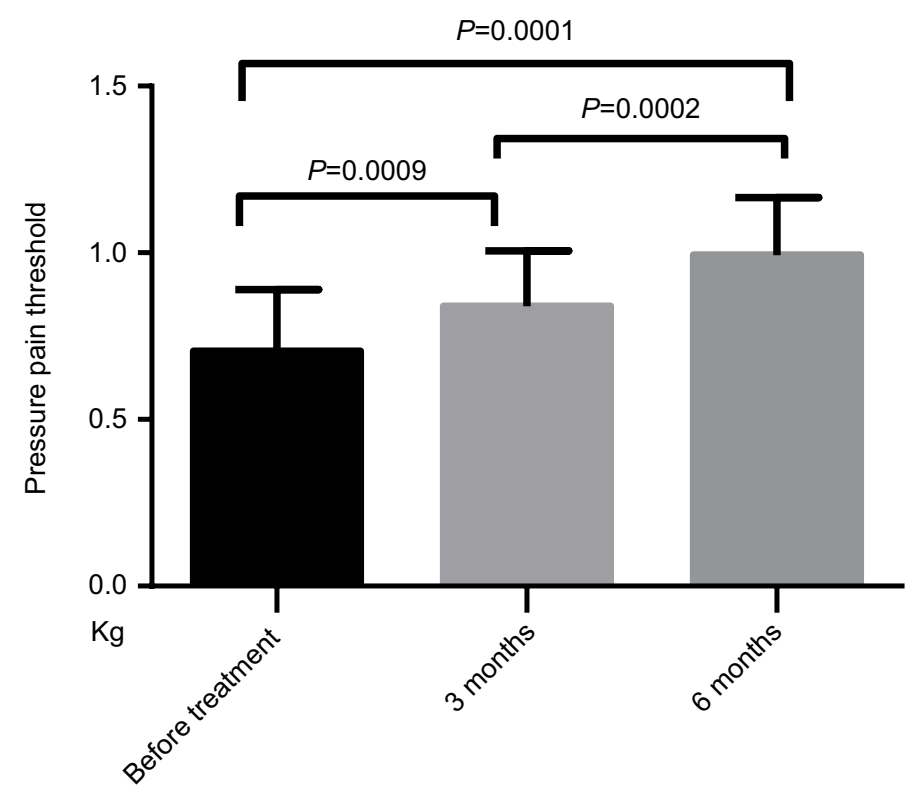

Figure 3 Changes in pressure pain threshold after topiramate treatment.

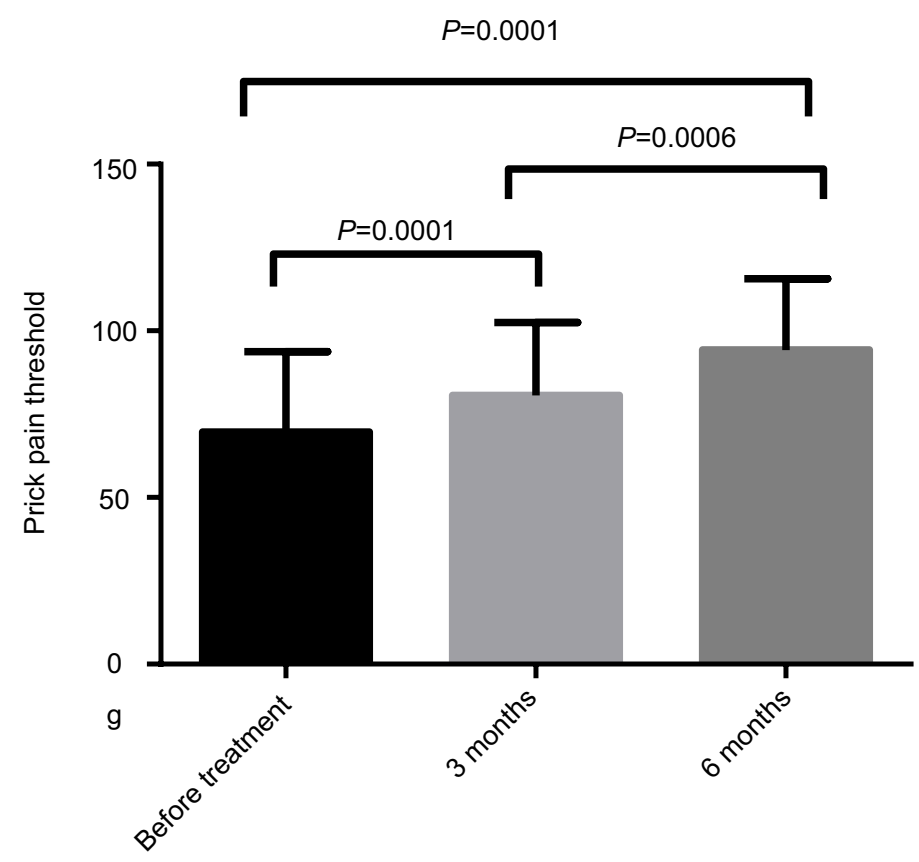

Figure $\mathbf{4}$ Changes in prick pain threshold after topiramate treatment.

In a human model of capsaicin-induced trigeminal sensitization, the pain area altered significantly across the menstrual cycle: maximum during menstruation to a minimum at the luteal phase. CS is responsible for persistent headache and allodynia. ${ }^{26}$ Early therapy may interfere with the peripheral sensitization and CS, thereby preventing chronic headache and allodynia. Thus, the model had two goals: to terminate acute attacks and prevent subsequent occurrence of the attack.
Different classes of drugs have been used for migraine prophylaxis, and these drugs act by normalizing the neuronal excitability through the modulation of ionic channels and neurotransmitter systems. These drugs have also been shown to block the cortical-induced depression and prevent peripheral sensitization and/or CS. The frequency of attacks and severity of migraine in patients was reduced markedly after administering topiramate. Consequently, 


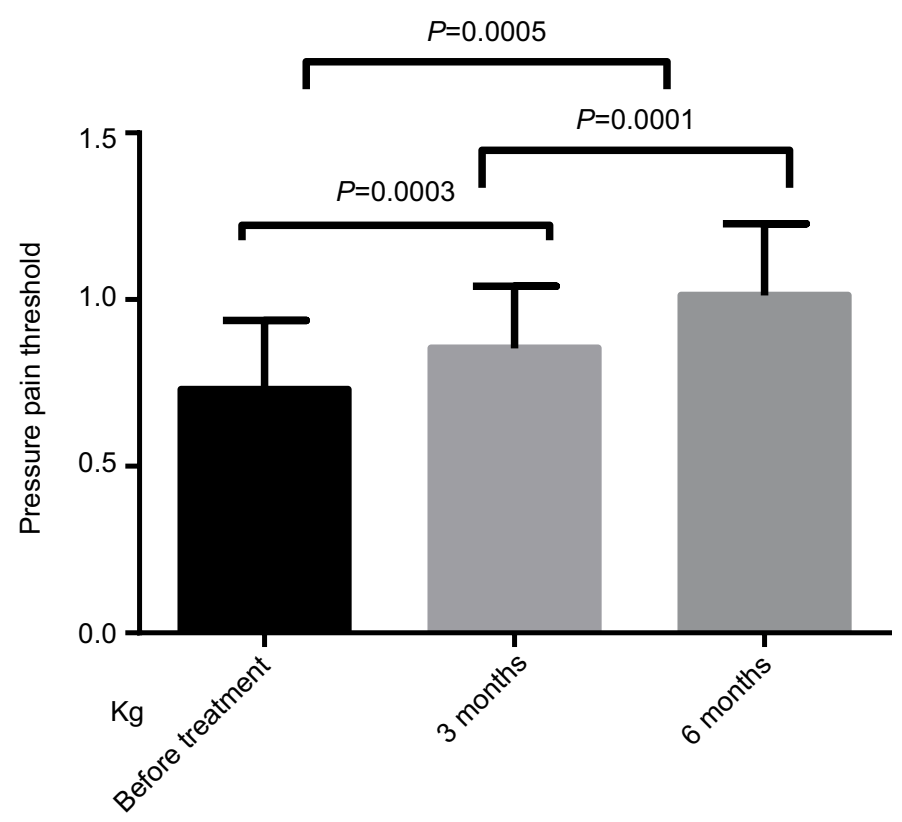

Figure 5 Changes in pressure pain threshold after flunarizine treatment.

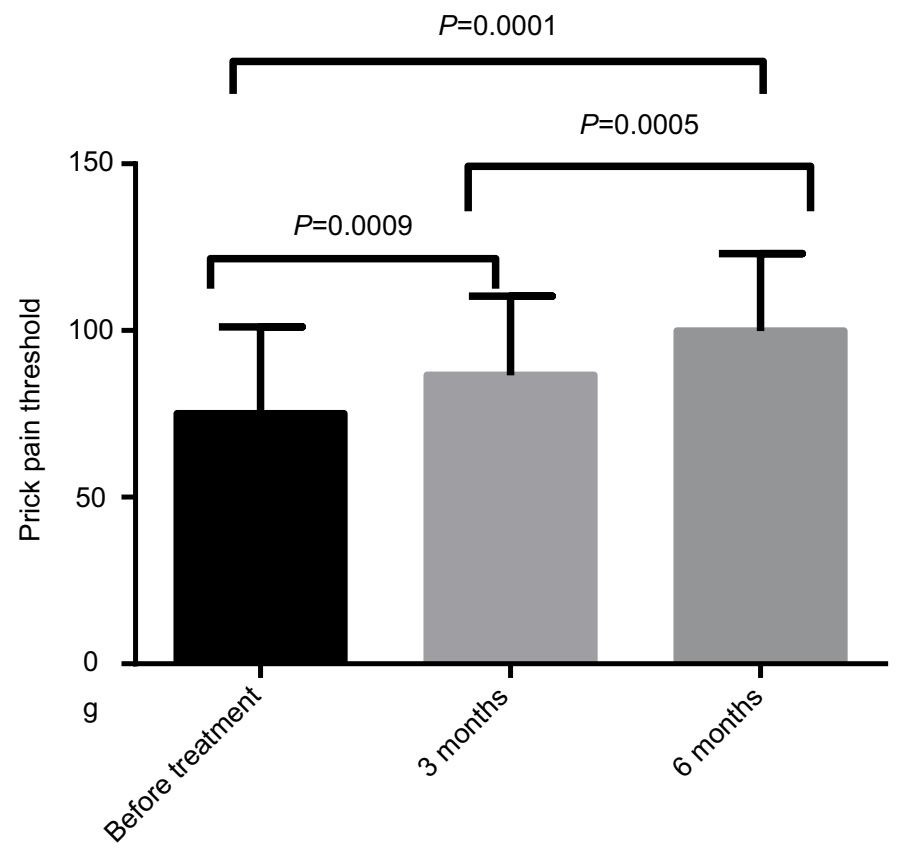

Figure 6 Changes in prick pain threshold after flunarizine treatment.

the duration of pain was shortened. Furthermore, Bussone et $\mathrm{al}^{27,28}$ demonstrated that topiramate was effective in the prevention of migraine based on three large randomized controlled trials $(P<0.001)$. The common significant dose-dependent adverse effects of topiramate included paresthesia and memory and concentration problems. The dosage of drugs was relatively lower, $50-100 \mathrm{mg} / \mathrm{d}$. Only three cases had paresthesia that was relieved after dose adjustment; no obvious adverse effects were noted during the follow-up visit.

The current study found that the thresholds of pressure and pricking were improved after administering flunarizine, 
Table I Changes in pain threshold before and after flunarizine and topiramate treatment

\begin{tabular}{|l|l|l|l|}
\hline Variables & $\begin{array}{l}\text { Flunarizine } \\
\text { group } \\
\text { (23 cases) }\end{array}$ & $\begin{array}{l}\text { Topiramate } \\
\text { group } \\
\text { (22 cases) }\end{array}$ & $\mathbf{P}$ \\
\hline Pressure pain (kg) & & & \\
Before treatment & $0.73 \pm 0.21$ & $0.70 \pm 0.18$ & 0.236 \\
3 months & $0.85 \pm 0.19$ & $0.83 \pm 0.17$ & 0.404 \\
6 months & $1.01 \pm 0.21$ & $0.99 \pm 0.17$ & 0.400 \\
Prick pain (g) & $75.06 \pm 26.06$ & $69.68 \pm 24.11$ & 0.057 \\
Before treatment & $86.59 \pm 23.67$ & $81.66 \pm 21.85$ & 0.138 \\
3 months & $99.80 \pm 23.25$ & $94.29 \pm 21.37$ & 0.160 \\
6 months & \multicolumn{3}{|l}{}
\end{tabular}

thereby designating it as an effective drug for relieving allodynia.

Flunarizine has been speculated as the first-line prophylaxis for migraine. However, the adverse effects of flunarizine include weight gain and drowsiness. In the current study, three cases showed weight gain that was relieved by reducing the dosage of the drug. Although one case exhibited rapid depression, the pain was relieved distinctly.

\section{Disclosure}

The authors report no conflicts of interest in this work.

\section{References}

1. Lipton RB, Stewart WF, Diamond S, Diamond ML, Reed M. Prevalence and burden of migraine in the United States: data from the American Migraine Study II. Headache. 2001;41(7):646-657.

2. Stewart W, Lipton R, Celentano D, et al. Prevalence of migraine headache in the United States: relation to age, income, race, and other sociodemographic factors. JAMA. 1991;267:64-69.

3. Bigal ME, Lipton RB. The epidemiology, burden, and comorbidities of migraine. Neurol Clin. 2009;27(2):321-334.

4. Burton WN, Conti DJ, Chen CY, Schultz AB, Edington DW. The economic burden of lost productivity due to migraine headache: a specific worksite analysis. J Occup Environ Med. 2002;44(6):523-529.

5. Bigal ME, Ashina S, Burstein R, et al. Prevalence and characteristics of allodynia in headache sufferers: a population study. Neurology. 2008;70(17):1525-1533.

6. Lipton RB, Bigal ME, Ashina S, et al. Cutaneous allodynia in the migraine population. Ann Neurol. 2008;63(2):148-158.

7. Lovati C, D'Amico D, Bertora P. Allodynia in migraine: frequent random association or unavoidable consequence? Expert Rev Neurother. 2009;9(3):395-408.

8. Burstein R, Yarnitsky D, Goor-Aryeh I, Ransil BJ, Bajwa ZH. An association between migraine and cutaneous allodynia. Ann Neurol. 2000;47(5):614-624.
9. Burstein R, Cutrer MF, Yarnitsky D. The development of cutaneous allodynia during a migraine attack clinical evidence for the sequential recruitment of spinal and supraspinal nociceptive neurons in migraine. Brain. 2000;123(8):1703-1709.

10. Aguggia M. Allodynia and migraine. Neurol Sci. 2012;33(Suppl 1):9-11.

11. Mark A. Louter, Johanneke E. Bosker, Willebrordus PJ, et al. Cutaneous allodynia as a predictor of migraine chronification. Brain. 2013:1-8.

12. Haut SR, Bigal ME, Lipton RB. Chronic disorders with episodic manifestations: focus on epilepsy and migraine. Lancet Neurol. 2006;5(2):148-157.

13. Ottman R, Lipton RB, Ettinger AB, et al. Comorbidities of epilepsy: results from the Epilepsy Comorbidities and Health (EPIC) survey. Epilepsia. 2011;52(2):308-315.

14. Winawer MR, Connors R, EPGP Investigators. Evidence for a shared genetic susceptibility to migraine and epilepsy. Epilepsia. 2013;54(2):288-295.

15. Gazerani P, Andersen OK, Arendt-Nielsen L. A human experimental capsaicin model for trigeminal sensitization. Gender-specific differences. Pain. 2005;118(1-2):155-163.

16. Brandes JL, Saper JR, Diamond M, et al. MIGR-002 Study Group. Topiramate for migraine prevention: a randomized controlled trial JAMA. 2004;291(8):965-973.

17. Miljanich GP, Ramachandran J. Antagonists of neuronal calcium channels: structure, function, and therapeutic implications. Annu Rev Pharmacol Toxicol. 1995;35:707-734.

18. Reveiz-Herault L, Cardona AF, Ospina EG, Carrillo P. Effectiveness of flunarizine in the prophylaxis of migraine: a meta-analytical review of the literature. Rev Neurol. 2003;36(10):907-912.

19. Headache Classification Subcommittee of the International Headache Society. The international classification of headache disorders: 2nd edition. Cephalalgia. 2004;24(Suppl 1):9-160.

20. Güven H, Çilliler AE, Çomoğlu SS. Cutaneous allodynia in patients with episodic migraine. Neurol Sci. 2013;34(8):1397-1402.

21. Palmeira C, Ashmawi HA, Posso I. Sex and pain perception and analgesia. Braz J Anesthesiol. 2011;61(6):814-828.

22. Matos R, Wang K, Jensen JD, et al. Quantitative sensory testing in the trigeminal region: site and gender differences. J Orofac Pain. 2011;25(2): 161-169.

23. Blau JN, Dexter SL. The site of pain origin during migraine attacks. Cephalalgia. 1981;1(3):143-147.

24. Katsarava Z, Schneeweiss S, Kurth T, et al. Incidence and predictors for chronicity of headache in patients with episodic migraine. Neurology. 2004;62(5):788-790.

25. Filatova E, Latysheva N, Kurenkov A. Evidence of persistent central sensitization in chronic headaches: a multi-method study. $J$ Headache Pain. 2008;9(5):295-300.

26. Burstein R, Levy D, Jakubowski M. Effects of sensitization of trigeminovascular neurons to triptan therapy during migraine. Rev Neurol. 2005;161(6-7):658-660.

27. Bussone G, Diener HC, Pfeil J, Schwalen S. Topiramate $100 \mathrm{mg} /$ day in migraine prevention: a pooled analysis of double-blind randomised controlled trials. Int J Clin Pract. 2005;59(8):961-968.

28. Brandes JL, Kudrow DB, Rothrock JF, et al. Assessing the ability of topiramate to improve the daily activities of patients with migraine. Mayo Clin Proc. 2006;81(10):1311-1319. 
The Journal of Pain Research is an international, peer reviewed, open access, online journal that welcomes laboratory and clinical findings in the fields of pain research and the prevention and management of pain. Original research, reviews, symposium reports, hypothesis formation and commentaries are all considered for publication.
The manuscript management system is completely online and includes a very quick and fair peer-review system, which is all easy to use. Visit http://www.dovepress.com/testimonials.php to read real quotes from published authors. 